\title{
The Sargasso Sea Eel Expedition 1979
}

\author{
F.-W. Tesch \\ Biologische Anstalt Helgoland (Zentrale); \\ Notkestr. 31, D-2000 Hamburg 52, Federal Republic of Germany
}

\begin{abstract}
The background and aims of the Sargasso Sea Expedition for exploring the reproduction of the eel (Anguilla spp.) in the North Atlantic are described. The ships used for the investigations in the Sargasso Sea and during transects across the North Atlantic were F. R. V. "Anton Dohrn" and R. V. "Friedrich Heincke", provided by the Federal Republic of Germany. Their technical and scientific equipment and their staffs are described. The courses of the cruises are mapped and the geographical positions of the investigations as well as the different instruments employed are tabulated as a source of basic data for more specialized papers published in this issue or elsewhere. Reference is also given to relevant papers which have been published elsewhere. Important but unpublished preliminary results are mentioned. Suggestions for future research needs are made.
\end{abstract}

\section{INTRODUCTION}

The 1979 Sargasso Sea Eel Expedition was initiated by a resolution of the Eel Symposium 1976 in Helsinki and organized by the International Council for the Exploration of the Sea (ICES) and the European Inland Fishery Advisory Commission (EIFAC) of the FAO. A planning group was established by the ICES to organize an international multiship expedition, but it proved impossible for different countries to contribute shipping time at appropriate dates. For this reason, the international project was split into a series of national cruises to the Sargasso Sea.

The first expedition on a national basis was undertaken in spring 1979 by two ships of the Federal Republic of Germany. One, the F. R. V. "Anton Dohrn", was sponsored by the DWK (German Scientific Commission for the Exploration of the Sea); the other, R. V. "Friedrich Heincke", is attached to the Biologische Anstalt Helgoland which organized the expedition.

\section{SCIENTIFIC BACKGROUND AND MAJOR OBJECTIVES}

The classical work by J. Schmidt (1924) in the early 1900's on the life history of European and American eels (Anguilla anguilla and $A$. rostrata) produced indirect evidence that the European eel spawns in the Sargasso Sea and suggested also that the spawning area for the American eel was to the southwest. These spawning areas were apparently attained by active migration of the adults, whereas the larvae were presumed to drift with major North Atlantic current systems to repopulate European and American waters. 
Spawning or maturing eels have not been caught in the Sargasso Sea or anywhere off the continents. The spawning area of the European eel has been roughly defined on the basis of small larvae, but no similar collections are available for the American eel. Naturally spawned eel eggs are unknown, as are migration routes, spawning conditions, behaviour and spawning times of the adults. The same is true for movements, species separation, feeding and growth of the larvae.

It is obvious that one or two cruises could not resolve all these questions. The investigations of the two German research vessels, therefore, were in the aid of reconnaissance. The major objective was to study the problems of sampling techniques and egg and larval identification as recommended by the ICES planning group. Other important samplings and measurements could be performed as well, such as oceanographic studies in the Sargasso Sea at that time of the year, the plankton environment and experimental investigations of the movement of adult (hormone-treated) eels. In addition, the collections with different equipment presented an opportunity to study other groups of animals and plants for various purposes.

\section{TECHNICAL AND SCIENTIFIC EQUIPMENT}

\section{Fishery Research Vessel "Anton Dohrn"}

Type: Stern trawler; length: $74 \mathrm{~m}$; displacement (cwl): 2,283 $\mathrm{t}$; power: 1,680 kw.

Scientific instruments used during the cruise: Multisonde; Hydrocast; Expendable Bathythermograph (XBT); Midwater trawl, 1,600 meshes circumference (MT 1,600); Isaacs Kidd Midwater Trawl (IKMT), opening $6 \mathrm{~m}^{2}$, meshsize $500 \mu \mathrm{m}$ with time-depth recorder; Multiple Opening and Closing Net and Environmental Factor Sensing System (MOCNESS), opening $1 \mathrm{~m}^{2}$, meshsize $350 \mu \mathrm{m}$; Multiple Closing Net (MCN) opening $0.25 \mathrm{~m}^{2}$, meshsize $350 \mu \mathrm{m}$; Neuston Net $335 \mu \mathrm{m}$.

\section{Research Vessel "Friedrich Heincke"}

Type: Stern trawler; length $38 \mathrm{~m}$; displacement (cwl): $466 \mathrm{t}$; power: $676 \mathrm{kw}$.

Scientific instruments used during the cruise: Hydrocast; Niskin bottles for plankton collections; Expendable Bathythermograph (XBT); Midwater trawl, 650 meshes circumference (MT 650); Isaacs Kidd Midwater Trawl (IKMT), opening $2 \mathrm{~m}^{2}$, meshsize $850 \mu \mathrm{m}$ with time depth recorder and pinger; Multiple Closing Net (MCN), opening $0.25 \mathrm{~m}^{2}$, meshsize $100 \mu \mathrm{m}$; Apstein net $55 \mu \mathrm{m}$; Neuston net $335 \mu \mathrm{m}$; Ultrasonic receiver for tracking eels and for depth recording of gear.

\section{Scientific staff}

\section{F. R. V. "Anton Dohrn"}

Scientist in charge: Dr. F.-W. Tesch (19/III - 9/V), Biologische Anstalt Helgoland, Hamburg (BAH).

Oceanography: G. Wegner (19/III - 9/V), Deutsche Wissenschaftliche Kommission für Meeresforschung, Hamburg; P. Wöckel, Deutsches Hydrographisches Institut, Hamburg. 
Systematics and sorting of Anguilliformes larvae: Dr. J. Boëtius (19/III - 17/IV), Danmark Fiskeri- og Havundersøgelser, Charlottenlund; Dr. A. Comparini (19/III - 17/ $\mathrm{IV}_{\text {; }}$ identification of species by electrophoresis), Istituto di Biologia Animale, Universita degli Studi di Padua; Dr. G. Peters (19/III - 17/IV), Institut für Hydrobiologie und Fischereiwissenschaft, Universität Hamburg; M. Schoth (19/III - 9/V), BAH; Dr. D. G. Smith (19/III - 17/IV), Marine Biomedical Institute, University of Texas, Galveston.

Predation of fishes on leptocephali: S. Appelbaum (19/III - 9/V), BAH.

Systematics of fish larvae from neuston and opening and closing nets: Dr. H. C. John $(19 / \mathrm{III}-9 / \mathrm{V})$, BAH.

Systematics of adolescent to adult fishes: Dr. E. Bertelsen (18/IV - 9/V), Zoologisk Museum, Copenhagen; Dr. R. H. Gibbs (19/III - 17/IV), Department of Vertebrate Zoology, National Museum of Natural History, Washington, D. C.; Dr. P. A. Hulley (18/IV - 9/V), South African Museum, Capetown; Dr. C. Karrer (19/III - 9/V), Zoologisches Institut, Universität Hamburg; Dr. G. Krefft (18/IV - 9/V), Institut für Seefischerei, Hamburg (ISF); Dr. J. Nielsen (18/IV - 9/V), Zoologisk Museum, Copenhagen; Dr. A. Post (18/TV - 9/V), ISF.

Systematics of Amphipods: Dr. H. G. Andres (19/III - 9/V), BAH.

Fungi of the seawater and of the Sargassum algae: Dr. A. Ulken (19/III - 17/IV), Institut für Meeresforschung, Bremerhaven.

Technical Assistance: J. Gomon (19/III - 9/V), Department of Vertebrate Zoology, National Museum of Natural History, Washington, D. C.; T. Minde (19/III - 17/IV) BAH; F. Ullah (18/IV - 9/V), ISF; U. Viebach (18/IV - 9/V), ISF.

\section{R. V. "Friedrich Heincke"}

Scientist in charge: Dr. F.-W. Tesch (12/II - 15/III), BAH; Prof. Dr. D. Schnack (16/III - 28/III), Institut für Hydrobiologie und Fischereiwissenschaft, Universität Hamburg; Dr. K.-H. Moebus (29/III - 26/IV), BAH.

Plankton investigations: R. Böttger (29/III - 18/IV), BAH; Dr. S. Muzavor (29/III 9/IV), Instituto Universitario dos Acores, Ponta Delgada.

Systematics and sorting of Anguilliformes larvae, tracking of adult eels: R. Kracht (12/II - 26/IV), BAH.

Technical Assistance: K.-L. Lange (21/II - 15/III), BAH.

\section{CRUISE REPORT}

In order to perform the Sargasso Sea program, F. R. V. "Anton Dohrn" left Bermuda on April 9 at the beginning of the second part of cruise No. 210/92. During the first part of its cruise the ship had been engaged in herring investigations in the N. W. Atlantic. The second part was divided into two sections. The first began and ended at Bermuda and included 7 North-South and one East-West transects through the presumed spawning area of Anguilla anguilla (Fig. 2). The second section crossed the eastern part of the eel spawning area once again and then the North Atlantic in the direction of the Bay of Biscay (Fig. 1).

Our original intention was to pass well to the north of the Azores, so as to include the 


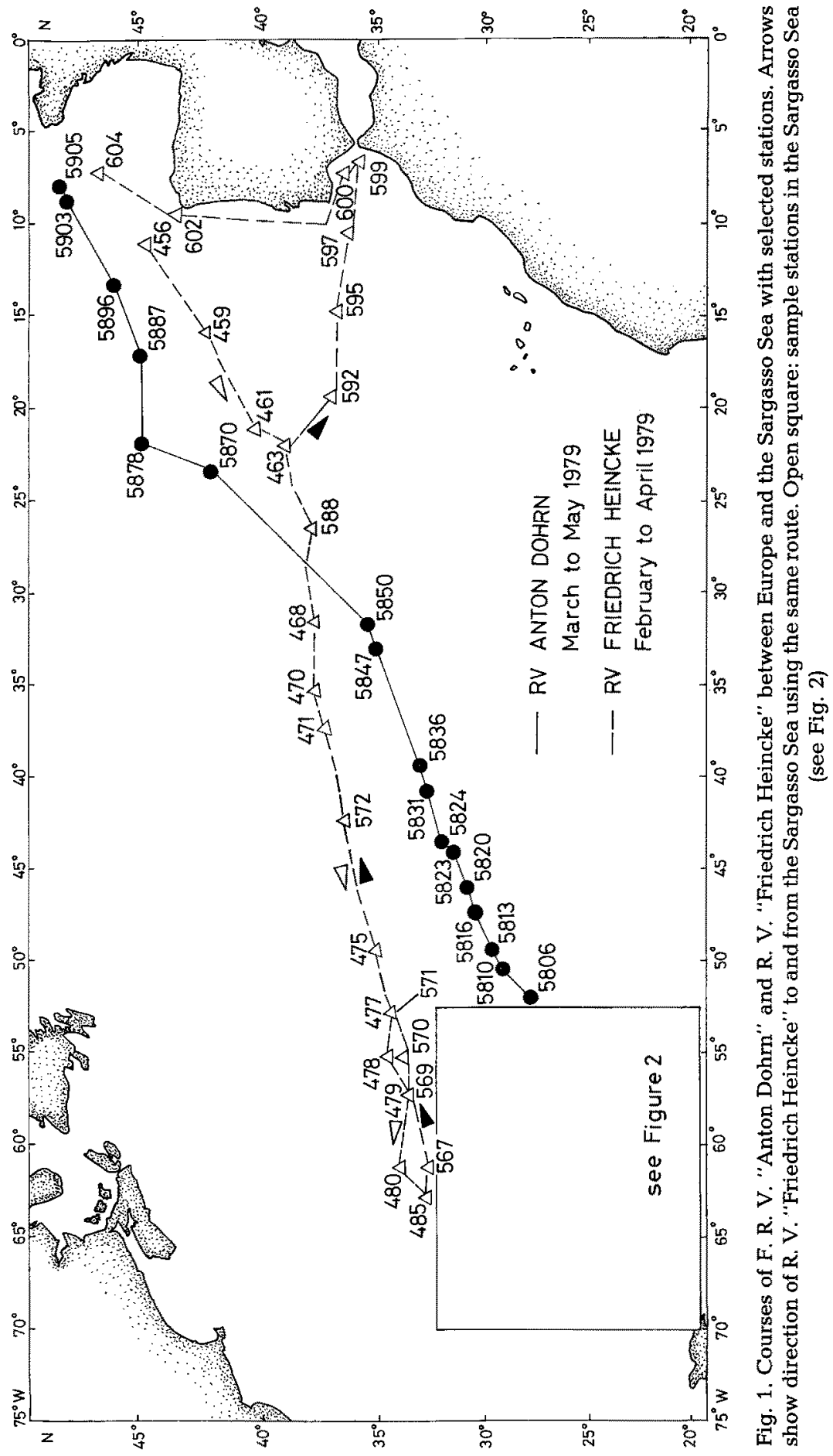




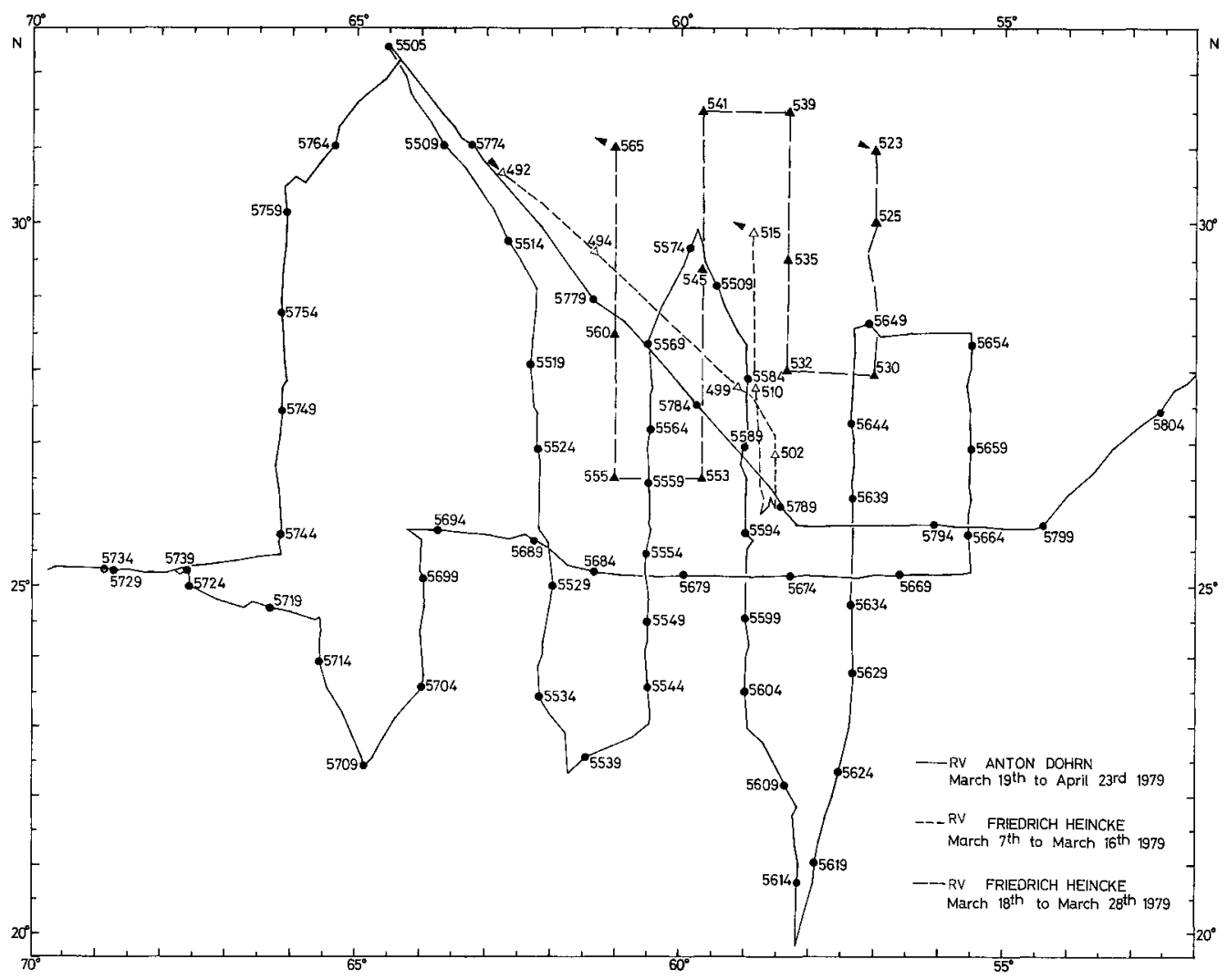

Fig. 2. Network of stations and routes of F. R. V. "Anton Dohrn" and R. V. "Friedrich Heincke" in the Sargasso Sea. The numbered black circles indicate each fifth station during the cruise of F. R. V. "Anton Dohrn"; the white and black triangles selected stations and the arrows the course of R. V.

"Friedrich Heinckes'" to and from Bermuda to the sample stations

"North Atlantic drift". But because of an accident to a crew member the ship had to call at the southern Azores Island St. Miguel. The transect therefore covered the region south of the "North Atlantic drift", but a stretch of 400 miles could not be investigated because permission had not been obtained for the 200 mile limit of the Azores.

The weather conditions were generally good except for two to three days in the Sargasso Sea area. A few hauls had to be cancelled, which had only a slight effect on the continuity of IKMT transects in the northern central Sargasso Sea near Bermuda. The geographic positions of the sampling stations with equipment used is presented in Table 1. XBT Stations which took place at least every two hours are not included.

The two transects made by R. V. "Friedrich Heincke" across the North Atlantic are shown in Figure 1, and the network of stations in the Sargasso Sea in Figure 2. The cruise was divided into seven phases with different compositions of the scientific staff in each. During the whole cruise beyond the continental shelf, eel larvae were sampled with the Isaacs Kidd Midwater Trawl, and bacteriophages with a microbiological bottle; hydrocasts and XBT measurements were made. 
Table 1a. Station list of F.R.V. "Anton Dohrn" Cruise No. 210/92, Part 2 (Sargasso Sea)

\begin{tabular}{|c|c|c|c|c|c|c|c|c|c|c|c|}
\hline $\begin{array}{c}\text { Station } \\
\text { No. }\end{array}$ & $\begin{array}{c}\text { Date } \\
1979 \\
\text { (day/ } \\
\text { month) }\end{array}$ & $\begin{array}{c}\text { Time } \\
\text { GTM } \\
-5 \mathrm{~h} \\
\text { (local } \\
\text { time) }\end{array}$ & $\begin{array}{l}\text { Lati- } \\
\text { tude } \\
\left({ }^{\circ} \mathrm{N}\right)\end{array}$ & $\begin{array}{l}\text { Longi- } \\
\text { tude } \\
\left({ }^{\circ} \mathrm{W}\right)\end{array}$ & $\begin{array}{l}\text { Multi- } \\
\text { sonde }\end{array}$ & $\begin{array}{l}\text { Neu- } \\
\text { ston }\end{array}$ & $\begin{array}{c}\text { IKMT } \\
\text { (haul } \\
\text { No./m } \\
\text { depth) }\end{array}$ & $\begin{array}{c}\text { MOC- } \\
\text { NESS } \\
\text { (haul } \\
\text { No.) }\end{array}$ & $\mathrm{MCN}$ & $\begin{array}{c}\text { Mid- } \\
\text { water } \\
\text { trawl } \\
\text { (m } \\
\text { depth) }\end{array}$ & $\begin{array}{c}\text { Sargas- } \\
\text { sum } \\
\text { collec- } \\
\text { tion }\end{array}$ \\
\hline 5507 & $20 / 3$ & 00.00 & $31^{\circ} 57^{\prime}$ & $64^{\circ} 16^{\prime}$ & 1 & 1 & & & 1 & & \\
\hline 5511 & $20 / 3$ & 09.00 & $30^{\circ} 24^{\prime}$ & $63^{\circ} 05^{\prime}$ & & 1 & & & & & \\
\hline 5512 & $20 / 3$ & 10.30 & $30^{\circ} 12^{\prime}$ & $62^{\circ} 55^{\prime}$ & & 1 & & & 1 & & \\
\hline 5513 & $20 / 3$ & 12.30 & $29^{\circ} 37^{\prime}$ & $62^{\circ} 35^{\prime}$ & 1 & & & & & & \\
\hline 5519 & $21 / 3$ & 01.30 & $28^{\circ} 06^{\prime}$ & $62^{\circ} 18^{\prime}$ & 1 & 1 & & & 1 & & \\
\hline 5522 & $21 / 3$ & 08.45 & $27^{\circ} 04^{\prime}$ & $62^{\circ} 14^{\prime}$ & & 2 & & & 1 & & \\
\hline 5526 & $21 / 3$ & 16.00 & $25^{\circ} 48^{\prime}$ & $62^{\circ} 10^{\prime}$ & 1 & 1 & & 1 & & 600 & \\
\hline 5527 & $21 / 3$ & 21.00 & $25^{\circ} 37^{\prime}$ & $61^{\circ} 56^{\prime}$ & & 2 & $1 / 70$ & & 1 & 80 & \\
\hline 5534 & $22 / 3$ & 09.00 & $23^{\circ} 25^{\prime}$ & $62^{\circ} 10^{\prime}$ & 1 & 1 & & 2 & 1 & & \\
\hline 5538 & $22 / 3$ & 19.55 & $22^{\circ} 21^{\prime}$ & $61^{\circ} 42^{\prime}$ & 1 & 2 & $2 / 130$ & 3 & 1 & & \\
\hline 5543 & $23 / 3$ & 08.55 & $23^{\circ} 13^{\prime}$ & $60^{\circ} 28^{\prime}$ & & 1 & & & 1 & & 1 \\
\hline 5550 & $23 / 3$ & 18.50 & $24^{\circ} 42^{\prime}$ & $60^{\circ} 30^{\prime}$ & 1 & 2 & $3 / 43$ & 4 & 1 & 150 & \\
\hline 5556 & $24 / 3$ & 08.00 & $25^{\circ} 53^{\prime}$ & $60^{\circ} 30^{\prime}$ & 1 & 1 & & 5 & 1 & & 1 \\
\hline 5558 & $24 / 3$ & 15.35 & $26^{\circ} 16^{\prime}$ & $60^{\circ} 30^{\prime}$ & 1 & & & & & & \\
\hline 5560 & $24 / 3$ & 18.17 & $26^{\circ} 35^{\prime}$ & $60^{\circ} 30^{\prime}$ & 1 & 1 & & & 1 & 600 & \\
\hline 5561 & $24 / 3$ & 21.23 & $26^{\circ} 42^{\prime}$ & $60^{\circ} 30^{\prime}$ & 1 & & & & & 150 & \\
\hline 5562 & $24 / 3$ & 22.52 & $26^{\circ} 51^{\prime}$ & $60^{\circ} 30^{\prime}$ & & 1 & $4 / 105$ & & & & \\
\hline 5565 & $25 / 3$ & 04.00 & $27^{\circ} 32^{\prime}$ & $60^{\circ} 29^{\prime}$ & 1 & & & & & & \\
\hline 5569 & $25 / 3$ & 09.00 & $28^{\circ} 21^{\prime}$ & $60^{\circ} 31^{\prime}$ & 1 & 1 & & & 1 & & \\
\hline 5572 & $25 / 3$ & 15.30 & $29^{\circ} 16^{\prime}$ & $60^{\circ} 03^{\prime}$ & & 1 & & & & & \\
\hline 5576 & $25 / 3$ & 20.00 & $29^{\circ} 53^{\prime}$ & $59^{\circ} 45^{\prime}$ & 1 & 1 & $5 / 100$ & & 1 & & \\
\hline 5582 & $26 / 3$ & 08.30 & $28^{\circ} 21^{\prime}$ & $59^{\circ} 59^{\prime}$ & 1 & 1 & & & 1 & & \\
\hline 5588 & $26 / 3$ & 17.30 & $27^{\circ} 02^{\prime}$ & $58^{\circ} 58^{\prime}$ & 1 & 3 & $6 / 100$ & 6 & 1 & 600 & \\
\hline 5594 & $27 / 3$ & 08.00 & $25^{\circ} 45^{\prime}$ & $59^{\circ} 00^{\prime}$ & 1 & 3 & & 7 & 1 & 1200 & 1 \\
\hline 5596 & $27 / 3$ & 18.00 & $25^{\circ} 32^{\prime}$ & $58^{\circ} 59^{\prime}$ & 1 & 2 & $7 / 148$ & 8 & 1 & 100 & \\
\hline 5600 & $28 / 3$ & 08.00 & $24^{\circ} 09^{\prime}$ & $59^{\circ} 00^{\prime}$ & 1 & 1 & & 9 & 1 & & \\
\hline 5602 & $28 / 3$ & 15.42 & $23^{\circ} 46^{\prime}$ & $58^{\circ} 59^{\prime}$ & & 1 & & & & 1200 & \\
\hline 5603 & $28 / 3$ & 18.30 & $23^{\circ} 37^{\prime}$ & $58^{\circ} 59^{\prime}$ & 1 & 2 & $8 / 88$ & & 1 & & \\
\hline 5605 & $28 / 3$ & 23.00 & $23^{\circ} 15^{\prime}$ & $58^{\circ} 59^{\prime}$ & 1 & & & & & & \\
\hline 5610 & $29 / 3$ & 08.00 & $21^{\circ} 50^{\prime}$ & $58^{\circ} 10^{\prime}$ & 2 & 2 & $9 / 130$ & & 1 & & 1 \\
\hline 5614 & $29 / 3$ & 17.15 & $20^{\circ} 45^{\prime}$ & $58^{\circ} 10^{\prime}$ & 1 & 1 & & & 1 & & \\
\hline 5615 & $29 / 3$ & 22.00 & $19^{\circ} 51^{\prime}$ & $58^{\circ} 10^{\prime}$ & 1 & & $10 / 90$ & & & & \\
\hline 5619 & $30 / 3$ & 08.00 & $21^{\circ} 03^{\prime}$ & $57^{\circ} 54^{\prime}$ & 1 & 2 & $11 / 150$ & & 1 & & \\
\hline 5623 & $30 / 3$ & 18.04 & $22^{\circ} 12^{\prime}$ & $57^{\circ} 35^{\prime}$ & & 2 & $12 / 150$ & & 1 & & \\
\hline 5627 & $31 / 3$ & 03.55 & $23^{\circ} 16^{\prime}$ & $57^{\circ} 21^{\prime}$ & & 1 & & & & 1800 & \\
\hline 5628 & $31 / 3$ & 08.35 & $23^{\circ} 31^{\prime}$ & $57^{\circ} 20^{\prime}$ & 1 & 2 & $13 / 185$ & & 2 & & 1 \\
\hline 5632 & $31 / 3$ & 17.25 & $24^{\circ} 23^{\prime}$ & $57^{\circ} 20^{\prime}$ & 1 & 3 & $14 / 100$ & & & 100 & \\
\hline 5638 & $01 / 4$ & 08.00 & $26^{\circ} 07^{\prime}$ & $57^{\circ} 22^{\prime}$ & 1 & 2 & $15 / 190$ & & 1 & & \\
\hline 5641 & $01 / 4$ & 14.40 & $26^{\circ} 44^{\prime}$ & $57^{\circ} 20^{\prime}$ & & 1 & & & & 600 & \\
\hline 5643 & $01 / 4$ & 17.30 & $27^{\circ} 01^{\prime}$ & $57^{\circ} 20^{\prime}$ & 1 & 2 & $16 / 100$ & 10 & 2 & & \\
\hline 5648 & $02 / 4$ & 08.00 & $28^{\circ} 44^{\prime}$ & $57^{\circ} 20^{\prime}$ & 1 & 1 & & & 1 & & \\
\hline 5652 & $02 / 4$ & 16.00 & $28^{\circ} 31^{\prime}$ & $56^{\circ} 00^{\prime}$ & & 2 & & & 1 & & \\
\hline 5653 & $02 / 4$ & 18.05 & $28^{\circ} 31^{\prime}$ & $55^{\circ} 30^{\prime}$ & 1 & & $17 / 75$ & & & & \\
\hline 5661 & $03 / 4$ & 08.00 & $26^{\circ} 23^{\prime}$ & $55^{\circ} 31^{\prime}$ & 1 & 2 & $18 / 185$ & & 1 & & 1 \\
\hline 5666 & $03 / 4$ & 17.10 & $25^{\circ} 12^{\prime}$ & $55^{\circ} 31^{\prime}$ & 1 & 4 & $19 / 90$ & 11 & 1 & 800 & \\
\hline 5670 & $04 / 4$ & 08.00 & $25^{\circ} 10^{\prime}$ & $57^{\circ} 00^{\prime}$ & 1 & 2 & $20 / 205$ & & 1 & & 1 \\
\hline 5674 & $04 / 4$ & 16.30 & $25^{\circ} 09^{\prime}$ & $58^{\circ} 07^{\prime}$ & 1 & 3 & $21 / 100$ & 12 & & 1200 & \\
\hline
\end{tabular}


Table 1a (continued)

\begin{tabular}{|c|c|c|c|c|c|c|c|c|c|c|c|}
\hline $\begin{array}{c}\text { Station } \\
\text { No. }\end{array}$ & $\begin{array}{c}\text { Date } \\
1979 \\
\text { (day/ } \\
\text { month) }\end{array}$ & $\begin{array}{c}\text { Time } \\
\text { GTM } \\
-5 \mathrm{~h} \\
\text { (local } \\
\text { time) }\end{array}$ & $\begin{array}{l}\text { Lati- } \\
\text { tude } \\
\text { ("N) }\end{array}$ & $\begin{array}{l}\text { Longi- } \\
\text { tude } \\
\left({ }^{\circ} \mathrm{W}\right)\end{array}$ & $\begin{array}{l}\text { Multi- } \\
\text { sonde }\end{array}$ & $\begin{array}{l}\text { Neu- } \\
\text { ston }\end{array}$ & $\begin{array}{l}\text { IKMT } \\
\text { (haul } \\
\text { No./m } \\
\text { depth) }\end{array}$ & $\begin{array}{l}\text { MOC- } \\
\text { NESS } \\
\text { (haul } \\
\text { No.) }\end{array}$ & $\mathrm{MCN}$ & $\begin{array}{c}\text { Mid- } \\
\text { water } \\
\text { trawl } \\
(\mathrm{m} \\
\text { depth) }\end{array}$ & $\begin{array}{l}\text { Sargas- } \\
\text { sum } \\
\text { collec- } \\
\text { tion }\end{array}$ \\
\hline 5679 & $05 / 4$ & 08.00 & $25^{\circ} 09^{\prime}$ & $59^{\circ} 56^{\prime}$ & 1 & 2 & $22 / 150$ & & & & \\
\hline 5684 & $05 / 4$ & 18.00 & $25^{\circ} 11^{\prime}$ & $61^{\circ} 19^{\prime}$ & 1 & 2 & $23 / 88$ & 13 & 1 & & \\
\hline 5688 & $06 / 4$ & 08.42 & $25^{\circ} 38^{\prime}$ & $62^{\circ} 15^{\prime}$ & 2 & 2 & $24 / 175$ & 14 & & & \\
\hline 5689 & $06 / 4$ & 18.28 & $25^{\circ} 38^{\prime}$ & $62^{\circ} 13^{\prime}$ & & 2 & $25 / 90$ & 15 & 1 & & \\
\hline 5695 & $07 / 4$ & 08.00 & $25^{\circ} 47^{\prime}$ & $64^{\circ} 12^{\prime}$ & 1 & 2 & $26 / 20$ & 16 & 1 & & 1 \\
\hline 5698 & $07 / 4$ & 18.35 & $25^{\circ} 13^{\prime}$ & $64^{\circ} 00^{\prime}$ & 1 & 2 & $27 / 110$ & & 1 & & \\
\hline 5702 & $08 / 4$ & 03.55 & $23^{\circ} 55^{\prime}$ & $63^{\circ} 38^{\prime}$ & & 1 & & & & 1800 & \\
\hline 5703 & $08 / 4$ & 08.10 & $23^{\circ} 42^{\prime}$ & $63^{\circ} 58^{\prime}$ & 1 & 2 & 28/195 & & 1 & & 1 \\
\hline 5709 & $08 / 4$ & 18.37 & $22^{\circ} 27^{\prime}$ & $64^{\circ} 51^{\prime}$ & 1 & 2 & $29 / 135$ & & 1 & & \\
\hline 5715 & $09 / 4$ & 08.00 & $24^{\circ} 15^{\prime}$ & $65^{\circ} 35^{\prime}$ & 1 & 2 & $30 / 180$ & 17 & 1 & & \\
\hline 5717 & $09 / 4$ & 16.55 & $24^{\circ} 33^{\prime}$ & $65^{\circ} 34^{\prime}$ & & 2 & $31 / 90$ & 18 & 1 & & \\
\hline 5720 & $10 / 4$ & 04.00 & $24^{\circ} 41^{\prime}$ & $66^{\circ} 20^{\prime}$ & & & & & & 1800 & \\
\hline 5721 & $10 / 4$ & 08.20 & $24^{\circ} 46^{\prime}$ & $66^{\circ} 37^{\prime}$ & 1 & 2 & $32 / 200$ & & 1 & & 1 \\
\hline 5722 & $10 / 4$ & 12.00 & $24^{\circ} 42^{\prime}$ & $66^{\circ} 45^{\prime}$ & & & & & & 1800 & \\
\hline 5724 & $10 / 4$ & 16.00 & $25^{\circ} 60^{\prime}$ & $67^{\circ} 33^{\prime}$ & & & & & & 1800 & \\
\hline 5725 & $10 / 4$ & 19.55 & $25^{\circ} 02^{\prime}$ & $67^{\circ} 38^{\prime}$ & 1 & 2 & $33 / 85$ & & 1 & & \\
\hline 5731 & $11 / 4$ & 08.00 & $25^{\circ} 15^{\prime}$ & $69^{\circ} 43^{\prime}$ & 1 & 2 & $34 / 200$ & & 1 & & 1 \\
\hline 5736 & $11 / 4$ & 19.00 & $25^{\circ} 13^{\prime}$ & $68^{\circ} 26^{\prime}$ & & 1 & & & & & \\
\hline 5738 & $12 / 4$ & 01.45 & $25^{\circ} 08^{\prime}$ & $67^{\circ} 39^{\prime}$ & & 2 & $35 / 90$ & 19 & & 1800 & \\
\hline 5738 & $12 / 4$ & 12.50 & $25^{\circ} 08^{\prime}$ & $67^{\circ} 39^{\prime}$ & 1 & 1 & $36 / 160$ & 20 & 1 & 1800 & \\
\hline 5738 & $12 / 4$ & 18.20 & $25^{\circ} 08^{\prime}$ & $67^{\circ} 39^{\prime}$ & & 1 & $37 / 90$ & $21 / 22$ & 1 & & \\
\hline 5743 & $13 / 4$ & 08.00 & $25^{\circ} 26^{\prime}$ & $66^{\circ} 10^{\prime}$ & 1 & 2 & $38 / 160$ & 23 & & & \\
\hline 5746 & $13 / 4$ & 18.45 & $26^{\circ} 12^{\prime}$ & $66^{\circ} 11^{\prime}$ & 1 & 2 & $39 / 85$ & 24 & 1 & & \\
\hline 5750 & $14 / 4$ & 08.00 & $27^{\circ} 46^{\prime}$ & $66^{\circ} 09^{\prime}$ & 1 & 2 & $40 / 200$ & & 1 & & \\
\hline 5756 & $14 / 4$ & 18.45 & $29^{\circ} 09^{\prime}$ & $66^{\circ} 10^{\prime}$ & 1 & 2 & $41 / 85$ & & 1 & & \\
\hline 5760 & $15 / 4$ & 04.05 & $30^{\circ} 27^{\prime}$ & $66^{\circ} 08^{\prime}$ & & 1 & & & & 1800 & \\
\hline 5761 & $15 / 4$ & 08.17 & $30^{\circ} 35^{\prime}$ & $65^{\circ} 59^{\prime}$ & 1 & 2 & $42 / 375$ & 25 & 1 & 1800 & \\
\hline 5764 & $15 / 4$ & 19.30 & $31^{\circ} 00^{\prime}$ & $65^{\circ} 22^{\prime}$ & 1 & & & & & & \\
\hline
\end{tabular}

The phases of the cruise are listed below, together with the special investigations: (1) Cuxhaven - Ponta Delgada (Azores) 12/II - 20/II، (2) Ponta Delgada - Bermuda 23/II 5/III, (3) Bermuda - Bermuda 7/III - 16/III (tracking of hormon treated eels, sampling for adult eels by midwater trawl), (4) Bermuda - Bermuda 18/III - 28/III (plankton and neuston investigations), (5) Bermuda - Ponta Delgada 31/III - 9/IV (plankton and neuston investigations), (6) Ponta Delgada - Portimao (Southportugal), (7) Portimao Cuxhaven.

Geographic positions of the different stations and equipment used are shown in Table 2. Only a few of the planned hauls of the IKMT during the transect had to be cancelled because of bad weather. Unfavourable weather conditions were encountered during the plankton investigations of the fourth phase, which caused some problems particularly for operations with the Multiple Closing Net. Generally, the weather in the northern central Sargasso Sea was more adverse than in the southern part so that the R. V. "Friedrich Heincke", operating in the northern area, had to work under more 
Table 1b. Station list F.R.V. "Anton Dohm" Cruise No. 210/92, Part 2 (Sargasso Sea - Bay of Biscay)

\begin{tabular}{|c|c|c|c|c|c|c|c|c|c|}
\hline $\begin{array}{l}\text { Station } \\
\text { No. }\end{array}$ & $\begin{array}{l}\text { Date } 1979 \\
\text { (day } / \\
\text { month) }\end{array}$ & $\begin{array}{l}\text { Local } \\
\text { time }\end{array}$ & GMT & $\begin{array}{c}\text { Latitude } \\
\text { ('N) }\end{array}$ & $\begin{array}{l}\text { Longitude } \\
\text { ("W) }\end{array}$ & $\begin{array}{l}\text { Multi- } \\
\text { sonde }\end{array}$ & $\begin{array}{l}\text { Neu- } \\
\text { ston }\end{array}$ & $\begin{array}{l}\text { IKMT } \\
\text { (haul No./ } \\
\text { m depth) }\end{array}$ & $\begin{array}{c}\text { Midwater } \\
\text { trawl } \\
\text { (m depth) }\end{array}$ \\
\hline 5772 & $19 / 4$ & 13.15 & $+4 h$ & $31^{\circ} 11^{\prime}$ & $63^{\circ} 32^{\prime}$ & 1 & 1 & & 1650 \\
\hline 5773 & $19 / 4$ & 19.30 & $+4 \mathrm{~h}$ & $31^{\circ} 15^{\prime}$ & $63^{\circ} 23^{\prime}$ & 1 & 1 & & 170 \\
\hline 5774 & $19 / 4$ & 20.30 & $+4 h$ & $31^{\circ} 01^{\prime}$ & $63^{\circ} 15^{\prime}$ & 1 & 1 & & 250 \\
\hline 5778 & $20 / 4$ & 05.30 & $+4 h$ & $29^{\circ} 54^{\prime}$ & $62^{\circ} 08^{\prime}$ & 1 & 1 & & \\
\hline 5780 & $20 / 4$ & 14.30 & $+4 h$ & $28^{\circ} 41^{\prime}$ & $60^{\circ} 59^{\prime}$ & 1 & 2 & & 2000 \\
\hline 5781 & $20 / 4$ & 21.00 & $+4 h$ & $28^{\circ} 20^{\prime}$ & $60^{\circ} 33^{\prime}$ & & 2 & $43 / 55$ & \\
\hline 5786 & $21 / 4$ & 09.00 & $+4 \mathrm{~h}$ & $26^{\circ} 46^{\prime}$ & $59^{\circ} 02^{\prime}$ & 1 & 1 & $44 / 380$ & \\
\hline 5788 & $21 / 4$ & 14.30 & $+4 \mathrm{~h}$ & $26^{\circ} 11^{\prime}$ & $58^{\circ} 26^{\prime}$ & 1 & 1 & & 1800 \\
\hline 5789 & $21 / 4$ & 18.20 & $+4 h$ & $26^{\circ} 06^{\prime}$ & $58^{\circ} 26^{\prime}$ & 1 & 2 & & \\
\hline 5790 & $21 / 4$ & 20.03 & $+4 \mathrm{~h}$ & $25^{\circ} 52^{\prime}$ & $58^{\circ} 12^{\prime}$ & & & $45 / 95$ & \\
\hline 5795 & $22 / 4$ & 09.00 & $+4 h$ & $25^{\circ} 51^{\prime}$ & $55^{\circ} 42^{\prime}$ & 1 & 1 & $46 / 208$ & \\
\hline 5797 & $22 / 4$ & 14.30 & $+4 \mathrm{~h}$ & $25^{\circ} 49^{\prime}$ & $54^{\circ} 48^{\prime}$ & 1 & 2 & & 2000 \\
\hline 5798 & $22 / 4$ & 20.05 & $+4 \mathrm{~h}$ & $25^{\circ} 49^{\prime}$ & $54^{\circ} 31^{\prime}$ & & 1 & $47 / 100$ & \\
\hline 5803 & $23 / 4$ & 09.25 & $+4 h$ & $27^{\circ} 12^{\prime}$ & $52^{\circ} 55^{\prime}$ & 1 & 1 & $48 / 200$ & \\
\hline 5805 & $23 / 4$ & 14.15 & $+4 \mathrm{~h}$ & $27^{\circ} 38^{\prime}$ & $52^{\circ} 22^{\prime}$ & 1 & 2 & & 2000 \\
\hline 5806 & $23 / 4$ & 19.00 & $+4 \mathrm{~h}$ & $27^{\circ} 49^{\prime}$ & $52^{\circ} 13^{\prime}$ & & 2 & $49 / 100$ & 250 \\
\hline 5810 & $24 / 4$ & 08.30 & $+4 \mathrm{~h}$ & $29^{\circ} 16^{\prime}$ & $50^{\circ} 30^{\prime}$ & 1 & 1 & $50 / 185$ & \\
\hline 5812 & $24 / 4$ & 15.00 & $+4 \mathrm{~h}$ & $29^{\circ} 40^{\prime}$ & $49^{\circ} 38^{\prime}$ & & 1 & & 2000 \\
\hline 5813 & $24 / 4$ & 17.50 & $+4 h$ & $29^{\circ} 41^{\prime}$ & $49^{\circ} 27^{\prime}$ & 1 & 3 & $51 / 95$ & 110 \\
\hline 5816 & $25 / 4$ & 09.00 & $+3 h$ & $30^{\circ} 22^{\prime}$ & $47^{\circ} 25^{\prime}$ & & 1 & $52 / 245$ & \\
\hline 5817 & $25 / 4$ & 16.00 & $+3 h$ & $30^{\circ} 43^{\prime}$ & $46^{\circ} 16^{\prime}$ & 1 & 2 & & 2000 \\
\hline 5818 & $25 / 4$ & 20.00 & $+3 h$ & $30^{\circ} 43^{\prime}$ & $46^{\circ} 16^{\prime}$ & & 1 & & 195 \\
\hline 5819 & $25 / 4$ & 21.00 & $+3 h$ & $30^{\circ} 47^{\prime}$ & $46^{\circ} 04^{\prime}$ & & 1 & & 310 \\
\hline 5820 & $25 / 4$ & 21.55 & $+3 h$ & $30^{\circ} 48^{\prime}$ & $46^{\circ} 02^{\prime}$ & & 1 & $53 / 40$ & \\
\hline 5823 & $26 / 4$ & 08.30 & $+3 h$ & $31^{\circ} 31^{\prime}$ & $44^{\circ} 02^{\prime}$ & 1 & 1 & $54 / 324$ & \\
\hline 5825 & $26 / 4$ & 15.50 & $+3 h$ & $31^{\circ} 51^{\prime}$ & $42^{\circ} 55^{\prime}$ & 1 & 3 & & 2000 \\
\hline 5826 & $26 / 4$ & 20.45 & $+3 h$ & $31^{\circ} 55^{\prime}$ & $42^{\circ} 46^{\prime}$ & & 1 & & 200 \\
\hline 5828 & $26 / 4$ & 21.46 & $+3 h$ & $31^{\circ} 56^{\prime}$ & $42^{\circ} 43^{\prime}$ & & 1 & $54 / 50$ & \\
\hline 5831 & $27 / 4$ & 08.30 & $+3 h$ & $32^{\circ} 45^{\prime}$ & $40^{\circ} 39^{\prime}$ & 1 & 1 & $55 / 235$ & \\
\hline 5833 & $27 / 4$ & 15.00 & $+3 h$ & $32^{\circ} 50^{\prime}$ & $39^{\circ} 49^{\prime}$ & & 2 & & 2000 \\
\hline 5834 & $27 / 4$ & 18.15 & $+3 h$ & $33^{\circ} 01^{\prime}$ & $39^{\circ} 34^{\prime}$ & 1 & 1 & $56 / 235$ & 185 \\
\hline 5835 & $27 / 4$ & 20.30 & $+3 h$ & $33^{\circ} 04^{\prime}$ & $39^{\circ} 29^{\prime}$ & & 1 & & 345 \\
\hline 5836 & $27 / 4$ & 21.34 & $+3 h$ & $33^{\circ} 04^{\prime}$ & $39^{\circ} 27^{\prime}$ & & & $57 / 40$ & \\
\hline 5839 & $28 / 4$ & 08.30 & $+2 h$ & $33^{\circ} 52^{\prime}$ & $37^{\circ} 11^{\prime}$ & 1 & 1 & & \\
\hline 5842 & $28 / 4$ & 16.30 & $+2 h$ & $34^{\circ} 21^{\prime}$ & $35^{\circ} 22^{\prime}$ & 1 & 2 & & 1300 \\
\hline 5843 & $28 / 4$ & 20.20 & $+2 h$ & $34^{\circ} 20^{\prime}$ & $35^{\circ} 24^{\prime}$ & & 1 & & 170 \\
\hline 5844 & $28 / 4$ & 21.20 & $+2 h$ & $34^{\circ} 21^{\prime}$ & $35^{\circ} 22^{r}$ & & 1 & & 320 \\
\hline 5847 & $29 / 4$ & 08.30 & $+2 \mathrm{~h}$ & $35^{\circ} 06^{\prime}$ & $33^{\circ} 09^{\prime}$ & 1 & 1 & $58 / 281$ & \\
\hline 5849 & $29 / 4$ & 16.20 & $+2 h$ & $35^{\circ} 24^{\prime}$ & $32^{\circ} 01^{\prime}$ & 1 & 2 & & 2000 \\
\hline 5850 & $29 / 4$ & 20.05 & $+2 h$ & $35^{\circ} 24^{\prime}$ & $31^{\circ} 53^{\prime}$ & & 2 & $59 / 35$ & 350 \\
\hline 5852 & $30 / 4$ & 06.00 & $+2 h$ & $35^{\circ} 20^{\prime}$ & $30^{\circ} 16^{\prime}$ & 1 & 1 & & 2000 \\
\hline 5855 & $30 / 4$ & 19.00 & $+2 h$ & $36^{\circ} 37^{\prime}$ & $28^{\circ} 13^{\prime}$ & 1 & & & \\
\hline 5861 & $01 / 5$ & 16.00 & $+1 \mathrm{~h}$ & $38^{\circ} 33^{\prime}$ & $24^{\circ} 50^{\prime}$ & 1 & & & \\
\hline 5864 & $02 / 5$ & 09.30 & $+1 \mathrm{~h}$ & $40^{\circ} 48^{\prime}$ & $23^{\circ} 59^{\prime}$ & 1 & & & \\
\hline 5865 & $02 / 5$ & 11.00 & $+1 \mathrm{~h}$ & $41^{\circ} 02^{\prime}$ & $23^{\circ} 52^{\prime}$ & & 1 & & 2000 \\
\hline 5867 & $02 / 5$ & 18.30 & $+1 \mathrm{~h}$ & $42^{\circ} 00^{\prime}$ & $23^{\circ} 32^{\prime}$ & 1 & 1 & & 1900 \\
\hline 5868 & $02 / 5$ & 19.10 & $+1 h$ & $42^{\circ} 05^{\prime}$ & $23^{\circ} 30^{\prime}$ & & 1 & & 200 \\
\hline 5869 & $02 / 5$ & 20.15 & $+1 \mathrm{~h}$ & $42^{\circ} 07^{\prime}$ & $23^{\circ} 30^{\prime}$ & & 1 & & 400 \\
\hline 5870 & $02 / 5$ & 21.56 & $+1 \mathrm{~h}$ & $42^{\circ} 08^{\prime}$ & $23^{\circ} 29^{\prime}$ & & 1 & $60 / 42$ & \\
\hline
\end{tabular}


Table $1 \mathrm{~b}$ (continued)

\begin{tabular}{|c|c|c|c|c|c|c|c|c|c|}
\hline $\begin{array}{c}\text { Station } \\
\text { No. }\end{array}$ & $\begin{array}{c}\text { Date } 1979 \\
\text { (day/ } \\
\text { month) }\end{array}$ & $\begin{array}{l}\text { Local } \\
\text { time }\end{array}$ & GMT & $\begin{array}{c}\text { Latitude } \\
\text { ('N] }\end{array}$ & $\begin{array}{l}\text { Longitude } \\
\text { ('W) }\end{array}$ & $\begin{array}{l}\text { Multi- } \\
\text { sonde }\end{array}$ & $\begin{array}{l}\text { Neu- } \\
\text { ston }\end{array}$ & $\begin{array}{c}\text { IKMT } \\
\text { (haul No. } \\
\text { m depth) }\end{array}$ & $\begin{array}{l}\text { Midwater } \\
\text { trawl } \\
\text { (m depth) }\end{array}$ \\
\hline 5873 & $03 / 5$ & 08.30 & & $43^{\circ} 53^{\prime}$ & $22^{\circ} 45^{\prime}$ & 1 & 1 & & \\
\hline 5875 & $03 / 5$ & 16.00 & & $44^{\circ} 54^{\prime}$ & $22^{\circ} 16^{f}$ & 1 & 2 & & 2000 \\
\hline 5876 & $03 / 5$ & 20.00 & & $44^{\circ} 56^{\prime}$ & $22^{\circ} 00^{\prime}$ & & & & 175 \\
\hline 5877 & $03 / 5$ & 21.00 & & $44^{\circ} 56^{\prime}$ & $21^{\circ} 57^{\prime}$ & & 1 & & 340 \\
\hline 5878 & $03 / 5$ & 21.36 & & $44^{\circ} 56^{\prime}$ & $21^{\circ} 55^{\prime}$ & & 1 & $61 / 45$ & \\
\hline 5881 & $04 / 5$ & 08.30 & & $44^{\circ} 58^{\prime}$ & $19^{\circ} 18^{\prime}$ & 1 & 1 & & \\
\hline 5884 & $04 / 5$ & 16.45 & & $44^{\circ} 55^{\prime}$ & $17^{\circ} 34^{\prime}$ & 1 & 1 & & 2000 \\
\hline 5885 & $04 / 5$ & 20.45 & & $44^{\circ} 55^{\prime}$ & $17^{\circ} 22^{\prime}$ & & 1 & & 205 \\
\hline 5886 & $04 / 5$ & 21.40 & & $44^{\circ} 56^{\prime}$ & $17^{\circ} 18^{\prime}$ & & 1 & & 350 \\
\hline 5887 & $04 / 5$ & 22.45 & & $44^{\circ} 56^{\prime}$ & $17^{\circ} 17^{\prime}$ & & & $62 / 50$ & \\
\hline 5889 & $05 / 5$ & 04.30 & & $45^{\circ} 00^{\prime}$ & $16^{\circ} 00^{\prime}$ & 1 & 1 & & \\
\hline 5890 & $05 / 5$ & 08.30 & & $45^{\circ} 14^{\prime}$ & $15^{\circ} 25^{\prime}$ & 1 & & & \\
\hline 5893 & $05 / 5$ & 16.25 & & $45^{\circ} 51^{\prime}$ & $13^{\circ} 42^{\prime}$ & 1 & 2 & & 2000 \\
\hline 5894 & $05 / 5$ & 20.33 & & $45^{\circ} 54^{\prime}$ & $13^{\circ} 30^{\prime}$ & & 1 & & 205 \\
\hline 5895 & $05 / 5$ & 21.13 & & $45^{\circ} 53^{\prime}$ & $13^{\circ} 27^{\prime}$ & & 1 & & 350 \\
\hline 5896 & $05 / 5$ & 22.17 & & $45^{\circ} 56^{\prime}$ & $13^{\circ} 25^{\prime}$ & & 1 & $63 / 38$ & \\
\hline 5898 & $06 / 5$ & 08.30 & & $46^{\circ} 44^{\prime}$ & $11^{\circ} 11^{\prime}$ & 1 & 1 & & \\
\hline 5900 & $06 / 5$ & 15.00 & & $47^{\circ} 23^{\prime}$ & $09^{\circ} 54^{\prime}$ & & 1 & & \\
\hline 5901 & $06 / 5$ & 18.00 & & $47^{\circ} 42^{\prime}$ & $09^{\circ} 08^{\prime}$ & & 1 & & 2000 \\
\hline 5902 & $06 / 5$ & 21.03 & & $47^{\circ} 45^{\prime}$ & $08^{\circ} 58^{\prime}$ & & 1 & $64 / 40$ & \\
\hline 5903 & $06 / 5$ & 22.15 & & $47^{\circ} 46^{\prime}$ & $08^{\circ} 56^{\prime}$ & 1 & & & \\
\hline 5904 & $07 / 5$ & 02.00 & & $47^{\circ} 58^{\prime}$ & $08^{\circ} 16^{\prime}$ & & & $65 / 40$ & \\
\hline
\end{tabular}

unfavourable conditions than the F. R. V. "Anton Dohrn" operating at the same time in the south.

\section{RESULTS AND FURTHER RESEARCH}

The results of the 1979 eel expedition, included in this issue of the journal "Helgoländer Meeresuntersuchungen", are mainly concerned with eel research; earlier publications and mimeographed papers of this matter are available by Comparini $(1980 \mathrm{a}, \mathrm{b})$, Comparini \& Rodino (1980), Kracht \& Tesch (1981), McCleave \& Harden Jones (1979), Schoth (1981), Stibane (1981), Tesch (1980a, b, 1982), Tesch et al. (1979), Wegner (1979a). Scientific results in other fields have been published by John (1982), Moebus (1980), Moebus \& Nattkemper (1981), Ulken (1980), Wegner (1979b). Additional material is currently being evaluated and will be published in this journal or elsewhere.

Promising but very preliminary results were obtained by treating silver eels with pituitary extracts on board and by tracking four of these specimens north of and in the centre of the spawning area of A. anguilla. The specimens could be observed swimming at a maximum depth of $700 \mathrm{~m}$ but also just below the surface. Three specimens showed a mean swimming direction of WSW; but one of these specimens, tracked in the central spawning area, exhibited a more random directional tendency. Technically, the Sargasso Sea area offered favourable conditions for tracking with ultrasonic methods because the thermo- and salinoclines were weak at that time of the year. 
Table 2. Station list of R.V. "Friedrich Heincke" Cruise No. 160

\begin{tabular}{|c|c|c|c|c|c|c|c|c|c|c|c|}
\hline $\begin{array}{l}\text { Station } \\
\text { No. }\end{array}$ & $\begin{array}{c}\text { Date } 1979 \\
\text { (day/month) }\end{array}$ & $\begin{array}{l}\text { Local } \\
\text { time }\end{array}$ & GMT & $\begin{array}{l}\text { Latitude } \\
\left({ }^{\circ} \mathrm{N}\right)\end{array}$ & $\begin{array}{l}\text { Longitude } \\
\left({ }^{\circ} \mathrm{W}\right)\end{array}$ & 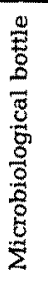 & 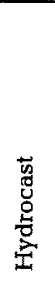 & 容 & 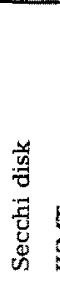 & 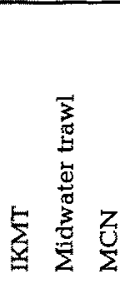 & 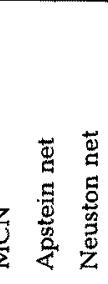 \\
\hline 454 & $15 / 2$ & $07.50-08.15$ & $-20^{\prime}$ & $47^{\circ} 43^{\prime}$ & $06^{\circ} 38^{\prime}$ & 1 & 1 & & & & \\
\hline 455 & $15 / 2$ & $10.40-11.20$ & $-20^{\prime}$ & $47^{\circ} 31^{\prime}$ & $07^{\circ} 00^{\prime}$ & & 1 & & & & \\
\hline 456 & $16 / 2$ & $08.15-14.20$ & $-20^{\prime}$ & $44^{\circ} 45^{\prime}$ & $10^{\circ} 55^{\prime}$ & 1 & 1 & 1 & & 2 & \\
\hline 457 & $16 / 2$ & $21.40-23.30$ & $-20^{\prime}$ & $44^{\circ} 07^{\prime}$ & $12^{\circ} 27^{\prime}$ & & & 1 & & 2 & \\
\hline 458 & $17 / 2$ & $09.00-09.45$ & $-20^{\prime}$ & $43^{\circ} 12^{\prime}$ & $14^{\circ} 17^{\prime}$ & 1 & 1 & 1 & 1 & & \\
\hline 459 & $17 / 2$ & $20.35-23.55$ & $-20^{\prime}$ & $42^{\circ} 25^{\prime}$ & $16^{\circ} 04^{\prime}$ & & & 1 & & 3 & \\
\hline 460 & $18 / 2$ & $09.25-10.25$ & $-20^{\prime}$ & $41^{\circ} 21^{\prime}$ & $18^{\circ} 04^{\prime}$ & 1 & 1 & & 1 & & \\
\hline 461 & $18 / 2$ & $21.00-00.25$ & $-20^{\prime}$ & $40^{\circ} 23^{\prime}$ & $20^{\circ} 09^{\prime}$ & & & & & 3 & \\
\hline 462 & $19 / 2$ & $10.00-11.00$ & $-20^{\prime}$ & $39^{\circ} 39^{\prime}$ & $21^{\circ} 48^{\prime}$ & 1 & 1 & & & & \\
\hline 463 & $19 / 2$ & $21.00-23.15$ & $-20^{\prime}$ & $39^{\circ} 00^{\prime}$ & $23^{\circ} 00^{\prime}$ & & & & & 2 & \\
\hline 464 & $20 / 2$ & $09.45-10.40$ & $-20^{\prime}$ & $38^{\circ} 00^{\prime}$ & $24^{\circ} 38^{\prime}$ & 1 & 1 & & & & \\
\hline 465 & $24 / 2$ & $08.00-08.10$ & $+2 \mathrm{~h}$ & $37^{\circ} 49^{\prime}$ & $29^{\circ} 03^{\prime}$ & 1 & & & & & \\
\hline 466 & $24 / 2$ & $10.25-11.00$ & $+2 h$ & $37^{\circ} 54^{\prime}$ & $29^{\circ} 18^{\prime}$ & & & & & 1 & \\
\hline 467 & $24 / 2$ & $17.00-18.30$ & $+2 h$ & $37^{\circ} 56^{\prime}$ & $30^{\circ} 30^{\prime}$ & & 1 & & 1 & & \\
\hline 468 & $24 / 2$ & $21.00-00.45$ & $+2 \mathrm{~h}$ & $37^{\circ} 50^{\prime}$ & $31^{\circ} 35^{\prime}$ & & & & & 3 & \\
\hline 469 & $25 / 2$ & $08.15-11.40$ & $+2 \mathrm{~h}$ & $37^{\circ} 58^{\prime}$ & $33^{\circ} 17^{\prime}$ & 1 & 1 & & & 1 & \\
\hline 470 & $25 / 2$ & $20.30-00.55$ & $+2 \mathrm{~h}$ & $37^{\circ} 40^{\prime}$ & $35^{\circ} 17^{\prime}$ & & & & & 3 & \\
\hline 471 & $26 / 2$ & $08.15-11.10$ & $+2 \mathrm{~h}$ & $37^{\circ} 20^{\prime}$ & $37^{\circ} 21^{\prime}$ & 1 & 1 & & & 1 & \\
\hline 472 & $27 / 2$ & $08.15-10.25$ & $+2 h$ & $36^{\circ} 33^{\prime}$ & $42^{\circ} 23^{t}$ & 1 & 1 & & & & \\
\hline 473 & $27 / 2$ & $20.30-23.45$ & $+2 \mathrm{~h}$ & $36^{\circ} 07^{\prime}$ & $44^{\circ} 42^{\prime}$ & & & & & 2 & \\
\hline 474 & $28 / 2$ & $08.15-12.05$ & $+3 h$ & $35^{\circ} 43^{\prime}$ & $47^{\circ} 05^{\prime}$ & 1 & 1 & & 1 & & \\
\hline 475 & $28 / 2$ & $20.15-23.45$ & $+3 \mathrm{~h}$ & $35^{\circ} 17^{\prime}$ & $49^{\circ} 30^{\prime}$ & & & & & 3 & \\
\hline 476 & $01 / 3$ & $08.15-13.50$ & $+3 h$ & $34^{\circ} 52^{\prime}$ & $51^{\circ} 31^{\prime}$ & 1 & 1 & & 1 & 3 & \\
\hline 477 & $01 / 3$ & $20.15-23.30$ & $+3 h$ & $34^{\circ} 29^{\prime}$ & $52^{\circ} 58^{\prime}$ & & & & & 2 & \\
\hline 478 & $02 / 3$ & $08.30-11.15$ & $+3 h$ & $34^{\circ} 01^{\prime}$ & $55^{\circ} 19^{\prime}$ & 1 & 1 & & 1 & 1 & \\
\hline 479 & $02 / 3$ & $20.30-23.45$ & $+3 \mathrm{~h}$ & $33^{\circ} 39^{\prime}$ & $57^{\circ} 22^{\prime}$ & & & & & 3 & \\
\hline 480 & $03 / 3$ & $08.30-09.15$ & $+3 \mathrm{~h}$ & $33^{\circ} 13^{\prime}$ & $59^{\circ} 20^{\prime}$ & 1 & 1 & & 1 & & \\
\hline 481 & $03 / 3$ & $18.15-19.35$ & $+3 \mathrm{~h}$ & $32^{\circ} 52^{\prime}$ & $61^{\circ} 10^{\prime}$ & & & & chni & lical test & \\
\hline 482 & $03 / 3$ & $20.20-23.40$ & $+3 h$ & $32^{\circ} 50^{\prime}$ & $61^{\circ} 20^{\prime}$ & & & & & 3 & \\
\hline 483 & $04 / 3$ & $00.35-02.10$ & $+3 \mathrm{~h}$ & $32^{\circ} 47^{\prime}$ & $61^{\circ} 38^{\prime}$ & & & & el tro & racking & \\
\hline 484 & $04 / 3$ & $08.30-10.40$ & $+3 \mathrm{~h}$ & $32^{\circ} 34^{\prime}$ & $62^{\circ} 57^{\prime}$ & 1 & 1 & & 1 & & \\
\hline 485 & $04 / 3$ & $10.50-14.10$ & $+3 h$ & $32^{\circ} 33^{\prime}$ & $63^{\circ} 01^{\prime}$ & & & & & 1 & \\
\hline 486 & $04 / 3$ & $20.45-21.50$ & $+3 h$ & $32^{\circ} 22^{\prime}$ & $64^{\circ} 29^{\prime}$ & & & & & 1 & \\
\hline 487 & $07 / 3$ & $21.15-23.15$ & $+4 h$ & $31^{\circ} 49^{\prime}$ & $65^{\circ} 09^{\prime}$ & & & & & 1 & \\
\hline 488 & $08 / 3$ & $00.12-06.00$ & $+4 h$ & $31^{\circ} 42^{\prime}$ & $65^{\circ} 25^{\prime}$ & & & & & 1 & \\
\hline 489 & $08 / 3$ & $09.25-09.30$ & $+4 \mathrm{~h}$ & $31^{\circ} 58^{\prime}$ & $65^{\circ} 11^{\prime}$ & 1 & & & & & \\
\hline 490 & $08 / 3$ & $10.55-15.30$ & $+4 \mathrm{~h}$ & $32^{\circ} 07^{\prime}$ & $65^{\circ} 00^{\prime}$ & & & & 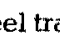 & racking & \\
\hline 491 & $08 / 3$ & $21.40-22.40$ & $+4 \mathrm{~h}$ & $31^{\circ} 44^{\prime}$ & $64^{\circ} 12^{\prime}$ & & & & & 1 & \\
\hline 492 & $09 / 3$ & $08.30-09.20$ & $+4 h$ & $30^{\circ} 40^{\prime}$ & $62^{\circ} 45^{\prime}$ & 1 & 1 & & 1 & & \\
\hline 493 & $09 / 3$ & 12.58 & $+4 h$ & $30^{\circ} 16^{\prime}$ & $62^{\circ} 10^{\prime}$ & & & 1 & & & \\
\hline 494 & $09 / 3$ & $18.10-19.00$ & $+4 \mathrm{~h}$ & $29^{\circ} 37^{\prime}$ & $61^{\circ} 20^{\prime}$ & & 1 & & & & \\
\hline 495 & $09 / 3$ & $20.00-22.20$ & $+4 \mathrm{~h}$ & $29^{\circ} 29^{\prime}$ & $61^{\circ} 09^{\prime}$ & & & & & 2 & \\
\hline 496 & $09 / 3$ & $23.00-06.00$ & $+4 h$ & $29^{\circ} 24^{\prime}$ & $61^{\circ} 03^{\prime}$ & & & & & 1 & \\
\hline 497 & $10 / 3$ & $09.25-09.30$ & $+4 h$ & $28^{\circ} 47^{\prime}$ & $60^{\circ} 21^{s}$ & 1 & & & & & \\
\hline
\end{tabular}


Table 2 (continued)

\begin{tabular}{|c|c|c|c|c|c|c|c|c|c|c|c|c|}
\hline $\begin{array}{l}\text { Station } \\
\text { No. }\end{array}$ & $\begin{array}{c}\text { Date } 1979 \\
\text { (day/month) }\end{array}$ & $\begin{array}{l}\text { Local } \\
\text { time }\end{array}$ & GMT & $\begin{array}{l}\text { Latitude } \\
\text { (N) }\end{array}$ & $\begin{array}{l}\text { Longitude } \\
\left({ }^{\circ} \mathrm{W}\right)\end{array}$ & 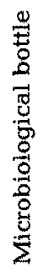 & 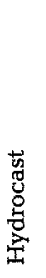 & $\vec{n}$ & 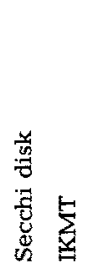 & 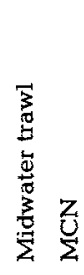 & 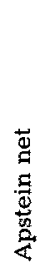 & 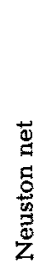 \\
\hline 498 & $10 / 3$ & $18.00-18.50$ & $+4 \mathrm{~h}$ & $27^{\circ} 47^{\prime}$ & $59^{\circ} 07^{\prime}$ & & 1 & & & & & \\
\hline 499 & $10 / 3$ & $20.15-22.05$ & $+4 \mathrm{~h}$ & $27^{\circ} 38^{\prime}$ & $58^{\circ} 53^{\prime}$ & & & & 2 & & & \\
\hline 500 & $10 / 3$ & $22.25-06.00$ & $+4 \mathrm{~h}$ & $27^{\circ} 35^{\prime}$ & $58^{\circ} 52^{\prime}$ & & & & & 1 & & \\
\hline 501 & $11 / 3$ & $07.30-07.35$ & $+4 \mathrm{~h}$ & $27^{\circ} 07^{\prime}$ & $58^{\circ} 33^{\prime}$ & 1 & & & & & & \\
\hline 502 & $11 / 3$ & $08.55-09.05$ & $+4 h$ & $26^{\circ} 53^{\prime}$ & $58^{\circ} 31^{\prime}$ & & & 1 & 1 & & & \\
\hline \multirow[t]{2}{*}{503} & $11 / 3$ & $13.54-$ & $+4 \mathrm{~h}$ & $26^{\circ} 05^{\prime}$ & $58^{\circ} 34^{\prime}$ & & & & & & & \\
\hline & $12 / 3$ & -05.30 & $+4 \mathrm{~h}$ & $26^{\circ} 03^{\prime}$ & $58^{\circ} 35^{\prime}$ & & & & el track & king & & \\
\hline 504 & $12 / 3$ & $07.20-11.00$ & $+4 h$ & $26^{\circ} 06^{\prime}$ & $58^{\circ} 35^{\prime}$ & 1 & & & & 1 & & \\
\hline 505 & $12 / 3$ & $11.40-19.15$ & $+4 h$ & $26^{\circ} 14^{\prime}$ & $58^{\circ} 36^{\prime}$ & & 1 & & & 1 & & \\
\hline 506 & $12 / 3$ & $19.25-23.45$ & $+4 h$ & $26^{\circ} 01^{\prime}$ & $58^{\circ} 47^{\prime}$ & & & & 4 & & & \\
\hline 507 & $13 / 3$ & $00.00-06.00$ & $+4 \mathrm{~h}$ & $26^{\circ} 05^{\prime}$ & $58^{\circ} 40^{\prime}$ & & & & & 1 & & \\
\hline 508 & $13 / 3$ & $07.30-07.45$ & $+4 \mathrm{~h}$ & $26^{\circ} 25^{\prime}$ & $58^{\circ} 47^{\prime}$ & 1 & & & 1 & & & \\
\hline 509 & $13 / 3$ & $10.45-11.30$ & $+4 \mathrm{~h}$ & $26^{\circ} 55^{\prime}$ & $58^{\circ} 47^{\prime}$ & & 1 & & & & & \\
\hline 510 & $13 / 3$ & $18.15-18.50$ & $+4 \mathrm{~h}$ & $27^{\circ} 44^{\prime}$ & $58^{\circ} 52^{\prime}$ & & & & echnical & 1 test & & \\
\hline 511 & $13 / 3$ & 19.35 & $+4 \mathrm{~h}$ & $27^{\circ} 47^{\prime}$ & $58^{\circ} 50^{\prime}$ & & & 1 & & & & \\
\hline 512 & $14 / 3$ & 05.20 & $+4 \mathrm{~h}$ & $29^{\circ} 15^{\prime}$ & $58^{\circ} 53^{\prime}$ & & & 1 & & & & \\
\hline 513 & $14 / 3$ & $07.30-07.35$ & $+4 \mathrm{~h}$ & $29^{\circ} 37^{\prime}$ & $58^{\circ} 53^{\prime}$ & 1 & & & & & & \\
\hline 514 & $14 / 3$ & 07.40 & $+4 \mathrm{~h}$ & $29^{\circ} 37^{\prime}$ & $58^{\circ} 53^{\prime}$ & & & 1 & & & & \\
\hline 515 & $14 / 3$ & $09.40-10.20$ & $+4 \mathrm{~h}$ & $29^{\circ} 53^{\prime}$ & $58^{\circ} 53^{\prime}$ & & 1 & & 1 & & & \\
\hline 516 & $14 / 3$ & $20.00-22.30$ & $+4 \mathrm{~h}$ & $30^{\circ} 41^{\prime}$ & $60^{\circ} 48^{\prime}$ & & & & 2 & & & \\
\hline 517 & $14 / 3$ & $22.50-23.50$ & $+4 \mathrm{~h}$ & $30^{\circ} 35^{\prime}$ & $60^{\circ} 45^{\prime}$ & & & & & 1 & & \\
\hline 518 & $15 / 3$ & $07.30-07.35$ & $+4 \mathrm{~h}$ & $31^{\circ} 12^{\prime}$ & $62^{\circ} 01^{\prime}$ & 1 & & & & & & \\
\hline 519 & $15 / 3$ & $13.55-18.30$ & $+4 \mathrm{~h}$ & $31^{\circ} 41^{\prime}$ & $63^{\circ} 07^{\prime}$ & & & & el track & king & & \\
\hline 520 & $15 / 3$ & $20.00-23.35$ & $+4 \mathrm{~h}$ & $31^{\circ} 43^{\prime}$ & $63^{\circ} 15^{\prime}$ & & & & 3 & & & \\
\hline 521 & $16 / 3$ & $06.00-06.15$ & $+4 \mathrm{~h}$ & $32^{\circ} 20^{\prime}$ & $64^{\circ} 35^{\prime}$ & & & & 1 & & & \\
\hline 522 & $20 / 3$ & $08.00-08.10$ & $+4 \mathrm{~h}$ & $31^{\circ} 04^{\prime}$ & $57^{\circ} 18^{\prime}$ & 1 & & & & & & \\
\hline 523 & $20 / 3$ & $10.00-15.50$ & $+4 \mathrm{~h}$ & $31^{\circ} 00^{\prime}$ & $57^{\circ} 00^{\prime}$ & & 1 & 1 & & 2 & 1 & 1 \\
\hline 524 & $20 / 3$ & $18.50-20.45$ & $+4 \mathrm{~h}$ & $30^{\circ} 30^{\prime}$ & $57^{\circ} 00^{\prime}$ & & & & & 1 & & 1 \\
\hline 525 & $20 / 3$ & $23.15-01.00$ & $+4 \mathrm{~h}$ & $30^{\circ} 00^{\prime}$ & $57^{\circ} 00^{\prime}$ & & & 1 & & 1 & 1 & \\
\hline 526 & $21 / 3$ & $08.00-08.05$ & $+4 \mathrm{~h}$ & $29^{\circ} 36^{\prime}$ & $57^{\circ} 05^{\prime}$ & 1 & & & & & & \\
\hline 527 & $21 / 3$ & $10.45-12.40$ & $+4 \mathrm{~h}$ & $29^{\circ} 36^{\prime}$ & $57^{\circ} 15^{\prime}$ & & & & & & 4 & 1 \\
\hline 528 & $21 / 3$ & $15.40-17.20$ & $+4 h$ & $29^{\circ} 00^{\prime}$ & $57^{\circ} 00^{\prime}$ & & & & & 1 & & 1 \\
\hline 529 & $21 / 3$ & $20.00-21.40$ & $+4 \mathrm{~h}$ & $28^{\circ} 30^{\prime}$ & $57^{\circ} 00^{\prime}$ & & & & & & 2 & 1 \\
\hline 530 & $22 / 3$ & $01.30-02.40$ & $+4 h$ & $28^{\circ} 00^{\prime}$ & $57^{\circ} 00^{\prime}$ & & & 1 & & & 2 & 1 \\
\hline 531 & $22 / 3$ & $08.00-08.05$ & $+4 \mathrm{~h}$ & $27^{\circ} 56^{\prime}$ & $57^{\circ} 44^{\prime}$ & 1 & & & & & & \\
\hline 532 & $22 / 3$ & $13.00-15.00$ & $+4 \mathrm{~h}$ & $28^{\circ} 00^{\prime}$ & $58^{\circ} 20^{\prime}$ & & 1 & & & 1 & 1 & 1 \\
\hline 533 & $22 / 3$ & $19.05-20.30$ & $+4 \mathrm{~h}$ & $28^{\circ} 30^{\prime}$ & $58^{\circ} 20^{\prime}$ & & & 1 & & & 3 & 1 \\
\hline 534 & $22 / 3$ & $23.40-00.40$ & $+4 \mathrm{~h}$ & $29^{\circ} 00^{\prime}$ & $58^{\circ} 20^{\prime}$ & & & & & & 2 & 1 \\
\hline 535 & $23 / 3$ & $03.50-05.05$ & $+4 h$ & $29^{\circ} 30^{\prime}$ & $58^{\circ} 20^{\prime}$ & & 1 & & & & 2 & 1 \\
\hline 536 & $23 / 3$ & $08.45-10.05$ & $+4 \mathrm{~h}$ & $30^{\circ} 00^{\prime}$ & $58^{\circ} 20^{\prime}$ & 1 & & & & & 2 & 1 \\
\hline 537 & $23 / 3$ & $12.30-15.30$ & $+4 \mathrm{~h}$ & $30^{\circ} 30^{\prime}$ & $58^{\circ} 20^{\prime}$ & & & & & 2 & 2 & 1 \\
\hline 538 & $23 / 3$ & $18.45-20.00$ & $+4 h$ & $31^{\circ} 00$ & $58^{\circ} 20^{\prime}$ & & & & & & 2 & 1 \\
\hline 539 & $23 / 3$ & $22.45-01.00$ & $+4 h$ & $31^{\circ} 30^{\prime}$ & $58^{\circ} 20^{\prime}$ & & & & & 1 & 2 & 1 \\
\hline 540 & $24 / 3$ & $04.00-05.00$ & $+4 \mathrm{~h}$ & $31^{\circ} 29^{\prime}$ & $58^{\circ} 53^{\prime}$ & & & & 1 & & & \\
\hline
\end{tabular}


Table 2 (continued)

\begin{tabular}{|c|c|c|c|c|c|c|c|c|c|c|c|c|}
\hline $\begin{array}{l}\text { Station } \\
\text { No. }\end{array}$ & $\begin{array}{c}\text { Date } 1979 \\
\text { (day/montb) }\end{array}$ & $\begin{array}{l}\text { Local } \\
\text { time }\end{array}$ & GMT & $\begin{array}{l}\text { Latitude } \\
\text { ("N) }\end{array}$ & $\begin{array}{c}\text { Longitude } \\
\left({ }^{\circ} \mathrm{W}\right)\end{array}$ & 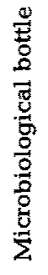 & 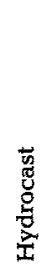 & 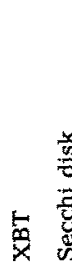 & 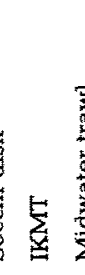 & 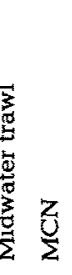 & 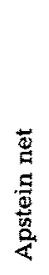 & 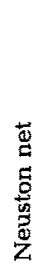 \\
\hline 541 & $24 / 3$ & $08.15-11.30$ & $+4 \mathrm{~h}$ & $31^{\circ} 30^{\prime}$ & $59^{\circ} 40^{\prime}$ & 1 & 1 & 1 & & 1 & 2 & 1 \\
\hline 542 & $24 / 3$ & $14.15-15.55$ & $+4 \mathrm{~h}$ & $31^{\circ} 00^{\prime}$ & $59^{\circ} 40^{\prime}$ & & & & & 1 & 1 & 1 \\
\hline 543 & $24 / 3$ & $18.10-19.40$ & $+4 \mathrm{~h}$ & $30^{\circ} 30^{\prime}$ & $59^{\circ} 40^{\prime}$ & & & 1 & & 1 & 1 & 1 \\
\hline 544 & $24 / 3$ & $22.30-23.50$ & $+4 \mathrm{~h}$ & $30^{\circ} 00^{\prime}$ & $59^{\circ} 40^{\prime}$ & & & 1 & & 1 & 1 & 1 \\
\hline 545 & $25 / 3$ & $02.35-03.45$ & $+4 \mathrm{~h}$ & $29^{\circ} 30^{\prime}$ & $59^{\circ} 40^{\prime}$ & & & & & 1 & 1 & 1 \\
\hline 546 & $25 / 3$ & $06.00-07.55$ & $+4 \mathrm{~h}$ & $29^{\circ} 00^{\prime}$ & $59^{\circ} 40^{\prime}$ & & & 1 & & 1 & 1 & 1 \\
\hline 547 & $25 / 3$ & $08.45-08.50$ & $+4 \mathrm{~h}$ & $28^{\circ} 50^{\prime}$ & $59^{\circ} 38^{\prime}$ & 1 & & & & & & \\
\hline 548 & $25 / 3$ & $10.30-11.45$ & $+4 \mathrm{~h}$ & $28^{\circ} 30^{\prime}$ & $59^{\circ} 40^{\prime}$ & & & 1 & & 1 & 1 & 1 \\
\hline 549 & $25 / 3$ & $14.30-15.55$ & $+4 \mathrm{~h}$ & $28^{\circ} 00^{\prime}$ & $59^{\circ} 40^{\prime}$ & & & & & 1 & 1 & 1 \\
\hline 550 & $25 / 3$ & $18.45-20.15$ & $+4 \mathrm{~h}$ & $27^{\circ} 30^{\prime}$ & $59^{\circ} 40^{\prime}$ & & & 1 & & 1 & 1 & 1 \\
\hline 551 & $25 / 3$ & $21.45-22.50$ & $+4 \mathrm{~h}$ & $27^{\circ} 13^{\prime}$ & $59^{\circ} 40^{\prime}$ & & & & 1 & & & \\
\hline 552 & $25 / 3$ & $23.35-00.50$ & $+4 \mathrm{~h}$ & $27^{\circ} 00^{\prime}$ & $59^{\circ} 40^{\prime}$ & & & 1 & & 1 & 1 & 1 \\
\hline 553 & $26 / 3$ & $03.30-06.15$ & $+4 \mathrm{~h}$ & $26^{\circ} 30^{\prime}$ & $59^{\circ} 40^{\prime}$ & & 1 & 1 & & 2 & 1 & 1 \\
\hline 554 & $26 / 3$ & $08.45-08.50$ & $+4 \mathrm{~h}$ & $26^{\circ} 31^{\prime}$ & $60^{\circ} 14^{\prime}$ & 1 & & & & & & \\
\hline 555 & $26 / 3$ & $12.35-15.50$ & $+4 \mathrm{~h}$ & $26^{\circ} 30^{\prime}$ & $61^{\circ} 00^{\prime}$ & & 1 & & & 2 & 1 & 1 \\
\hline 556 & $26 / 3$ & $18.20-19.44$ & $+4 \mathrm{~h}$ & $27^{\circ} 00^{\prime}$ & $61^{\circ} 00^{\prime}$ & & & 1 & & 1 & 1 & 1 \\
\hline 557 & $26 / 3$ & $19.53-21.40$ & $+4 \mathrm{~h}$ & $27^{\circ} 00^{\prime}$ & $61^{\circ} 00^{\prime}$ & & & & 3 & & & \\
\hline 558 & $26 / 3$ & $23.45-00.55$ & $+4 \mathrm{~h}$ & $27^{\circ} 30^{\prime}$ & $61^{\circ} 00^{\prime}$ & & & 1 & & 1 & 1 & 1 \\
\hline 559 & $27 / 3$ & $03.40-04.45$ & $+4 \mathrm{~h}$ & $28^{\circ} 00^{\prime}$ & $61^{\circ} 00^{\prime}$ & & & & & 1 & 1 & 1 \\
\hline 560 & $27 / 3$ & $07.40-09.05$ & $+4 \mathrm{~h}$ & $28^{\circ} 30^{\prime}$ & $61^{\circ} 00^{\prime}$ & 1 & & & & 1 & 1 & 1 \\
\hline 561 & $27 / 3$ & $12.00-13.25$ & $+4 h$ & $29^{\circ} 00^{\prime}$ & $61^{\circ} 00^{\prime}$ & & & & & 1 & 1 & 1 \\
\hline 562 & $27 / 3$ & $16.20-17.40$ & $+4 \mathrm{~h}$ & $29^{\circ} 30^{\prime}$ & $61^{\circ} 00^{\prime}$ & & & & & 1 & 1 & 1 \\
\hline 563 & $27 / 3$ & $20.30-23.10$ & $+4 h$ & $30^{\circ} 00^{\prime}$ & $61^{\circ} 00^{\prime}$ & & & 1 & 1 & 1 & 1 & 1 \\
\hline 564 & $28 / 3$ & $01.40-04.05$ & $+4 h$ & $30^{\circ} 30^{\prime}$ & $61^{\circ} 00^{\prime}$ & & & 1 & & 2 & 1 & 1 \\
\hline $565 a$ & $28 / 3$ & $06.45-09.55$ & $+4 h$ & $31^{\circ} 00^{\prime}$ & $61^{\circ} 00^{\prime}$ & 1 & 1 & 1 & & 2 & 1 & 1 \\
\hline $565 \mathrm{~b}$ & $31 / 3$ & $08.10-08.15$ & $+4 h$ & $32^{\circ} 20^{\prime}$ & $64^{\circ} 38^{\prime}$ & 1 & & & & & & \\
\hline 566 & $31 / 3$ & $16.45-18.55$ & $+3 h$ & $32^{\circ} 33^{\prime}$ & $63^{\circ} 01^{\prime}$ & & & 1 & 1 & 1 & & 1 \\
\hline 567 & $01 / 4$ & $02.00-03.35$ & $+3 h$ & $32^{\circ} 50^{\prime}$ & $61^{\circ} 20^{\prime}$ & & & 1 & 1 & 1 & & 1 \\
\hline 568 & $01 / 4$ & $09.15-09.20$ & $+3 h$ & $33^{\circ} 03^{\prime}$ & $59^{\circ} 59^{\prime}$ & 1 & & & & & & \\
\hline 569 & $01 / 4$ & $21.00-23.50$ & $+3 h$ & $33^{\circ} 39^{\prime}$ & $57^{\circ} 22^{\prime}$ & & & 1 & 2 & 1 & & 2 \\
\hline 570 & $02 / 4$ & $08.15-11.45$ & $+3 h$ & $34^{\circ} 01^{\prime}$ & $55^{\circ} 15^{\prime}$ & 1 & & 1 & 1 & 2 & & 1 \\
\hline 571 & $02 / 4$ & $22.35-00.00$ & $+3 h$ & $34^{\circ} 29^{\prime}$ & $52^{\circ} 58^{\prime}$ & & & & 1 & & & \\
\hline 572 & $05 / 4$ & $07.15-11.45$ & $+2 h$ & $36^{\circ} 03^{\prime}$ & $42^{\circ} 28^{\prime}$ & 1 & & & 1 & & & \\
\hline 573 & $05 / 4$ & $12.50-16.40$ & $+2 h$ & $36^{\circ} 09^{\prime}$ & $42^{\circ} 17^{\prime}$ & & & & & 2 & & 1 \\
\hline 574 & $05 / 4$ & $21.00-21.30$ & $+2 h$ & $36^{\circ} 15^{\prime}$ & $41^{\circ} 13^{\prime}$ & & & & & & & 1 \\
\hline 575 & $06 / 4$ & $03.00-03.30$ & $+2 h$ & $36^{\circ} 23^{\prime}$ & $40^{\circ} 08^{\prime}$ & & & & & & & 1 \\
\hline 576 & $06 / 4$ & $08.15-08.25$ & $+2 \mathrm{~h}$ & $36^{\circ} 26^{\prime}$ & $39^{\circ} 04^{\prime}$ & 1 & & & & & & \\
\hline 577 & $06 / 4$ & $09.00-09.40$ & $+2 h$ & $36^{\circ} 27^{\prime}$ & $38^{\circ} 52^{\prime}$ & & & & & & & 1 \\
\hline 578 & $06 / 4$ & $15.00-15.30$ & $+2 \mathrm{~h}$ & $36^{\circ} 31^{\prime}$ & $37^{\circ} 46^{\prime}$ & & & & & & & 1 \\
\hline 579 & $06 / 4$ & $21.00-21.30$ & $+2 \mathrm{~h}$ & $36^{\circ} 40^{\prime}$ & $36^{\circ} 35^{\prime}$ & & & & & & & 1 \\
\hline 580 & $07 / 4$ & $03.00-03.30$ & $+2 \mathrm{~h}$ & $36^{\circ} 53^{\prime}$ & $35^{\circ} 26^{\prime}$ & & & & & & & 1 \\
\hline 581 & $07 / 4$ & $08.15-08.25$ & $+2 h$ & $37^{\circ} 03^{\prime}$ & $34^{\circ} 21^{\prime}$ & 1 & & & & & & \\
\hline 582 & $07 / 4$ & $09.05-09.45$ & $+2 \mathrm{~h}$ & $37^{\circ} 04^{\prime}$ & $34^{\circ} 11^{\prime}$ & & & & & 1 & & 1 \\
\hline 583 & $07 / 4$ & $14.00-15.35$ & $+2 \mathrm{~h}$ & $37^{\circ} 09^{\prime}$ & $33^{\circ} 34^{\prime}$ & & & & & 2 & & 1 \\
\hline
\end{tabular}


Table 2 (continued)

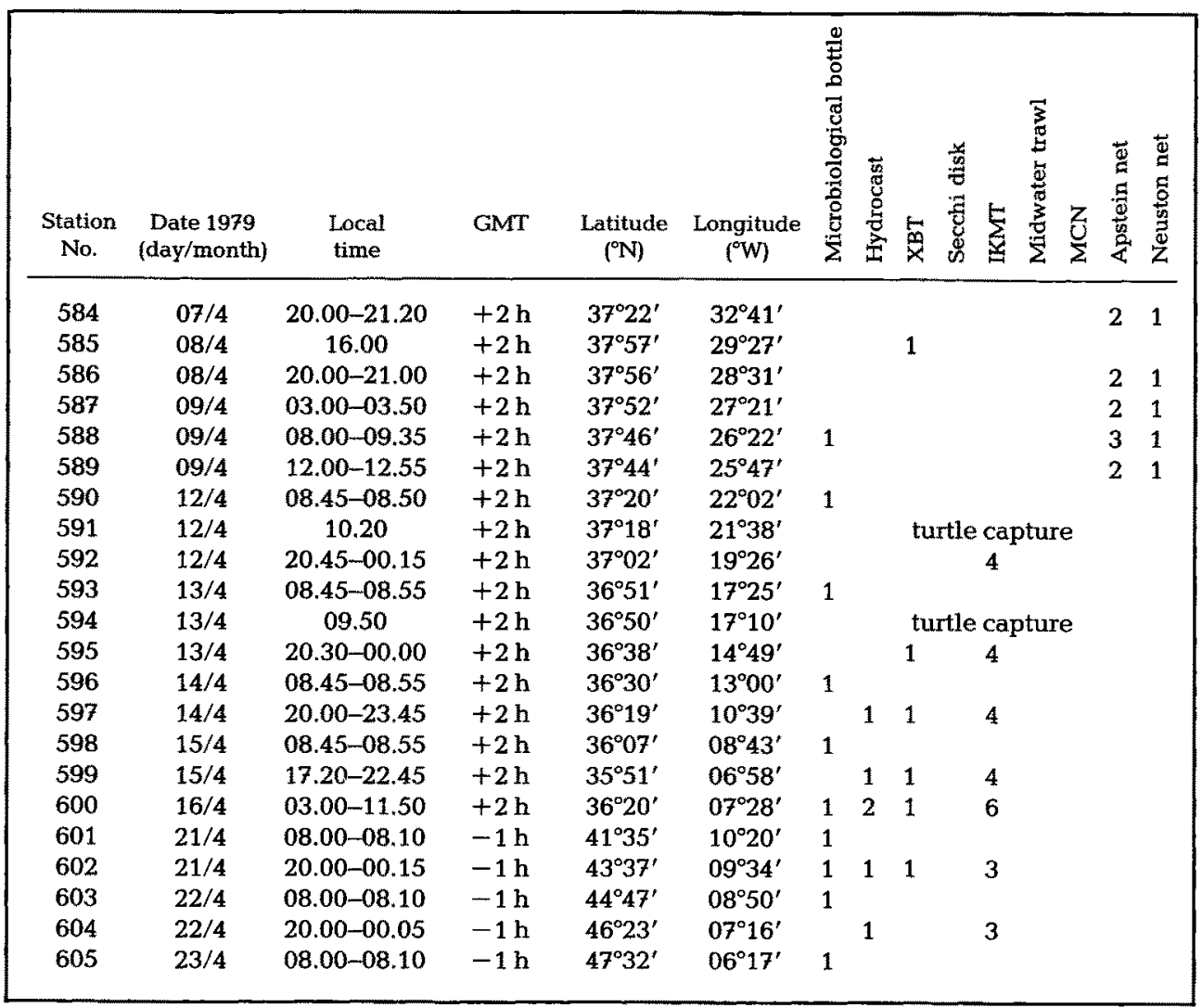

Besides other organisms, fish eggs were also sorted out from the IKMT catches (Table 1) simultaneously with the leptocephali. More than 7000 eggs were structurally classified (Stibane, 1981), but the taxonomic determination was very limited. Publication without detailed analysis is therefore unsuitable. It is very likely that in the sorted samples no eggs of Anguilla sp. were found but probably a great number of other anguilliform fish eggs $(23 \%)$. This coincides with the comparatively great numbers of anguilliform 0-group fish larvae, e.g. Serrivomer sp. and Nemichthys scolopaceus, in the spawning area of Anguilla sp. (Tesch et al., 1979).

As a consequence of the 1979 eel expedition, one additional transect across the North Atlantic for the collection of leptocephali was made in autumn 1979 by F. R. V. "Anton Dohrn" (Kracht \& Tesch, 1981; Kracht, 1982). The results of an earlier investigation involving collections of eel larvae along transects through the Gulf Stream are included in this issue (Kleckner \& McCleave, 1982).

In Spring 1981, there were two further cruises into the Sargasso Sea. One U. S. American and one German (FRG) ship, both collecting larvae, cooperated in order to cover the southeastern area of the Sargasso Sea and to investigate the beginning of the spawning season (Tesch, 1980a). One of the main objectives of R. V. "Friedrich Heincke" 
on its second visit to the Sargasso Sea was to investigate the patchiness of 0-group larvae occurrence. Two transects across the Atlantic were included, one of which covered the southern limits of I- and II-group larvae.

Discussions about future research on Atlantic eel reproduction (Tesch, 1980a) revealed that there is great need for further sampling of eel larvae over a wide area and over many months of the year. Considerations about the effort required to cover these wide areas disclosed that, at present, research is not possible because resources are insufficient. Since sampling is restricted by this limitation, the best solution would be to sample a smaller area intensively over a longer period of time. Oceanographic investigations in the presumed spawning area should be included. It is evident, especially since the 1981 expedition but also from evidence supplied by earlier cruises, that 0 -group larvae occur in patches which are more than 30 nautical miles in diameter. Analysis of these patches in regard to time and space, species and age composition is necessary. The investigation of the transatlantic migration of the larvae is also of major importance. This requires intensive sampling on the routes between the Sargasso Sea and Europe, Greenland and North America. Electrophoretic studies of 0-group larvae should be repeated. The search for adult eels has been unsuccessful. Trawl catches, therefore, should not be continued, but other techniques should be applied or developed, e.g. the tracking of hormonally treated eels, use of hormonally matured females as lures or the direct use of a possible eel pheromone, use of light as an attractant, and television.

Greater efforts are necessary if these questions are to be solved on a larger scale. For a better understanding of the biology of eel reproduction, a multiship activity (as internationally, originally planned) and many single, specially equipped cruises are indispensible. One step towards better fundamental knowledge of eel migration is the present international, intensive investigation of the North Atlantic circulation (ICES and SCOR).

Eel reproduction is regarded mainly as a problem of biological research. Sea fishery research which is in a position to carry out the considerable shipping effort and to apply the techniques needed for the aforementioned studies does not focus on eel research. Commercial sea fisheries exploit only a small part of the total amount of the world eel production. The total world yield of eel, according to the available statistics (FAO), amounts to ca. $100,000 \mathrm{t}$. The actual world yield, however, exceeds this amount; the statistics are not complete. With regard to its high commercial value, per total world yield, the eel ranks 6th-8th in importance when compared with other fish. In price, per unit weight, however, the eel occupies the second position after the salmon. This high yield is due mainly to the progress made in recent years in aquaculture, and the increase is far above average compared with the results gained with other fish. The supply of young fish for eel production is still provided by the exceptionally large natural resources (e.g. from the Sargasso Sea spawning area) and not yet by aquaculture. We, therefore, have good reason to intensify research activities on eel reproduction. This is not only necessary with respect to the surveillance of natural resources but also to increase our knowledge especially for developing artificial spawning methods.

Acknowledgements. The investigations carried out during and following the Sargasso Sea Eel Expedition 1979 were supported by the Bundesministerium für Forschung und Technologie (MFE0336), Bonn (FRG). 


\section{LITERATURE CITED}

Comparini، A., 1980a. Distributione dei genotipi della malico deidrogenasi (MDH) en un campione di leptocephali di Anguilla del Mar dei Sargasso. - Atti Ass. genet. ital. 25.

Comparini, A., 1980b. Variabilita enzimatica nelle anguilli. Primi dati su leptocephali di Anguilla anguilla (L.) e Anguilla rostrata (Lesueur). - Boll. Zool. (Suppl.) 46, 239-240.

Comparini, A. \& Rodino, E., 1980. Electrophoretic evidence for the two species of Anguilla leptocephali in the Sargasso Sea. - Nature, Lond. 287, 435-437.

John, H.-C., 1982. Distribution of Halobates micans (Heteroptera, Gerridae) in the Northern Atlantic during Spring, with comments on its catchability in neuston nets. - "Meteor" Forsch. Ergebn. (D), 34, 31-36.

Kleckner, R. C. \& McCleave, J. D., 1982. Entry of migrating American eel leptocephali into the Gulf Stream system. - Helgoländer Meeresunters. 35, 329-339.

Kracht, R. \& Tesch, F.-W., 1981. Progress report on the eel expedition of R. V. "Anton Dohrn" and R. C. "Friedrich Heincke" to the Sargasso Sea 1979. - Envir. Biol. Fish. 6, 311-375.

Kracht, R. 1982. On the geographic distribution and migration of I- and II-group eel larvae as studied during the 1979 Sargasso Sea Expedition. - Helgoländer Meeresunters. 35, 321-327.

McCleave, J. D. \& Harden Jones, F. R., 1979. Eels: new interest in an old problem. - Nature, Lond. $278,782-3$.

Moebus, K., 1980. A method for the detection of bacteriophages from ocean water. - Helgoländer Meeresunters. 34, 1-14.

Moebus, K. \& Nattkemper, H., 1981. Bacteriophage sensitivity patterns among bacteria isolated from marine waters. - Helgoländer Meeresunters. 34, 275-385.

Schmidt, J., 1924. The breeding places of the eel. - A. Rep. Smithsonian Instn 1924, 279-316.

Schoth, M., 1981. Die Verteilung der frühen Leptocephalusstadien des Atlantischen Aales der deutschen Sargassomeer-Expedition 1979. Dipl.-Arb., Univ. Hamburg, 103 pp. (unpubl.).

Stibane, F., 1981. Die Verteilung pelagischer Fischeier aus Fängen der deutschen SargassoseeExpedition 1979. Dipl.-Arb., Univ. Hamburg, 127 pp. (unpubl.).

Tesch, F.-W., 1980a. Report on the available data of the Atlantic Anguilla larvae, presented to the ICES Planning Group for the Atlantic Eel Expedition. - C.M./ICES/M 7, 1-10.

Tesch, F.-W., 1980b. Report of the international eel expedition planning group. - C. M./ICES/ $M 43,1-2$.

Tesch, F.-W., 1982. Neues aus der Aalforschung. - Fisch u. Fang 23, 606-608.

Tesch, F.-W., Kracht, R., Schoth, M., Smith, D. G. \& Wegner, G., 1979. Report on the eel expedition of FRV "Anton Dohrn" and R. K. "Friedrich Heincke" to the Sargasso Sea 1979. - C.M./ICES/ M 6, 1-29.

Ulken, A., 1980. Phycomycetenfunde in der Sargassosee. - Veröff. Inst. Meeresforsch. Bremerh. 18, $21-27$.

Wegner, G., 1979a. First hydrographic results from the Sargasso Sea expedition with F. R. V. "Anton Dohrn" 19. 3. to 9. 5. 1979. - C.M./ICES/C 5, 1-14.

Wegner, G., 1979b. F. R. V. "Anton Dohrn" XBT sections in the North Atlantic Ocean between January and May 1979. - Polymode News/70, 3-7. 